\title{
For Better or Forever: Formal versus Informal Enforcement
}

\author{
Joel Sobel, University of California, San Diego
}

This article contrasts supporting partnerships through relational contracting and supporting partnerships through formal legal institutions. A large population of players interact in bilateral relationships. Efficiency requires cooperation, but cheating yields a higher shortterm payoff. There is a positive probability that the maximum feasible payoff available to a partnership decreases. Opportunistic behavior makes it impossible to realize the efficient outcome. A legal system can lead to efficient contracting. Without such a system, productive relationships arise in equilibrium if it is costly to initiate new relationships. This type of relational contracting tends to make partnerships last longer than is efficient.

\section{Introduction}

All but the simplest transactions depend on trust. Enforceable contracts are just one way in which buyers can be assured that they will receive what they order and sellers can be assured that they will be paid. In some settings, formal contracts may be costly to write or difficult to enforce. People use informal means to make profitable trades.

This article examines an environment in which agents pair off to play a game. Opportunistic behavior in the game is attractive to an individual but is collectively inefficient. I contrast two ways to limit opportunistic behavior. The first method, relational contracting, uses standard argu-

Participation in discussions of University of California, San Diego's reading group in applied theory stimulated this article. Thanks to Dan Klerman, John McMillan, Joel Watson, and Chris Woodruff for useful ideas and corrections. I am grateful to the National Science Foundation for financial support. Contact author Joel Sobel at jsobel@ucsd.edu.

[Journal of Labor Economics, 2006, vol. 24, no. 2]

(C) 2006 by The University of Chicago Press. All rights reserved. 0734-306X/2006/2402-0003\$10.00 
ments from repeated games. If opportunistic behavior today leads to punishment tomorrow, then self-interested agents may forgo short-term gains in order to maintain the relationship. The second method relies on a legal system to enforce contracts that require cooperation.

In the model of this article, a large population of players interact in pairs. Initially, interactions take the form of a prisoner's dilemma. Maximizing joint payoffs requires cooperation, but cheating yields a higher short-term payoff. Established partnerships run the risk of growing stale. I model this by assuming that after each interaction there is a positive probability (independent across matches) that the maximum feasible payoff available to a partnership decreases. In a perfect world, agents would find partners without cost, cooperate until the relationship grew stale, and then, by mutual consent, break off the partnership in order to start new, more productive relationships. Opportunistic behavior may make it impossible to realize this outcome. In a world where every agent cooperates, it would be attractive for one agent to cheat, break off his relationship, and find a new partner to exploit. Opportunistic behavior will arise unless it is possible to detect and punish cheaters directly or it is costly to find new partners.

The model makes clear a qualitative difference between formal and relational contracting: improved enforcement leads to shorter relationships. When there is an effective legal system, starting new relationships is not risky. Provided that it is not hard to find a new partner, there is no reason to continue to participate in a partnership that has grown stale. When there is relational contracting, however, cooperation only arises in equilibrium when it is costly to start a new relationship. Without this cost, agents could behave opportunistically with impunity. ${ }^{1}$ When it is costly to start a new relationship, individuals have incentives to remain in existing relationships even when these have gone stale. Consequently, one expects (and one finds in the model) that the existence of an effective legal system leads to shorter, but more productive, partnerships. This finding is consistent with the work of McMillan and Woodruff (1999, 2000a), which provides a measure of persistence of relationships. Their surveys ask firms how they would respond to an offer by a new trading partner to supply goods at prices significantly below what they currently pay. Firms exhibit a reluctance to abandon their current supplier even if goods are available at lower prices elsewhere. My results give a simple theoretical explanation of the finding and suggest that a firm's "loyalty" should be inversely related to the effectiveness of formal enforcement mechanisms.

Relational contracts provide a substitute for formal enforcement. Although La Porta et al. (1998) find that growth is strongly linked to strong

${ }^{1}$ Proposition 1 states the result formally. 
financial and legal institutions, McMillan and Woodruff provide evidence that informal contracting plays a major role in Vietnam's development and Allen, Qian, and Qian (2005) attribute China's growth to the strength of private-sector enterprises that rely on relational contracting instead of formal enforcement. While I am not aware of cross-national evidence on the duration of trading partnerships, the model of this article suggests that internal relationships in economies that lack formal enforcement institutions will be more stable than those found in other economies.

Greif (1994) contrasts two institutions for supporting cooperation that parallel the formal and informal institutions modeled in this article. Greif contrasts the behavior of Maghribi traders, whose society was based on a collectivist culture that encouraged information sharing and group enforcement of norms, to the individualistic society of Genoa. The societies found different mechanisms to support successful trading within relationships that provided agents with an opportunity to make short-run gains through uncooperative behavior.

Greif models the strategic problem faced by merchants as a sequence of bilateral, one-sided prisoner's dilemma games. The strategic structure is slightly different from the model of this article, but the essential difference is that the value of stale relationships is zero (so that partnerships dissolve with fixed probability). Greif assumes that the collectivist culture permits information sharing. Consequently, the collectivist Maghribis shared knowledge about agents who had cheated in the past. These agents had trouble finding new partners. The Genoese were reluctant to share information and developed formal enforcement mechanisms because relational contracts were not dependable. Greif speculates that the individualistic society of Genoa led to efficiency gains by stimulating initiative. One source of these gains would be found in the ability to create new partnerships with a wide range of agents, which my model demonstrates is more likely in the presence of effective enforcement.

My model predicts that turnover in labor contracts increases with the quality of enforcement mechanisms. This finding is consistent with research but has not been modeled extensively. Jovanovic's (1979) influential model of job turnover derives several stylized properties of wage dynamics by assuming that employment relations form under incomplete information about the quality of the worker-employee match. In the course of an employment relationship, a worker-firm pair learns about the productivity of their partnership. Poor matches break up, so the average productivity of a worker increases with job tenure. In Jovanovic's model, workers are paid their marginal product, so wages increase with job tenure. Turnover rate is determined by the level of uncertainty and the speed at which workers and firms learn about the quality of matches. In my model, the quality of a partnership is known. Separations may arise due to a change in the partnership's quality. Turnovers increase when contracts are 
easier to enforce. My model also makes a prediction about how the productivity of a match depends on the length of the match. Since all relationships are productive in their initial stage but might decay, newly formed partnerships have higher productivity. ${ }^{2}$

The qualitative features of my model seem well suited to the marriage market. Couples have an opportunity to learn about the quality of their match during dating, so it is reasonable to assume that marriages can generate large surpluses at the beginning of a relationship. Interests and opportunities of marital partners change, however, and it may be in the best interest of marital partners to break up. The Coase theorem predicts that marriages will end when it is efficient to do so. Becker's (1981) model implies that divorces occur when the sum of the benefits to the partners in the marriage is less than their total outside opportunities. These theories predict that a change in divorce laws will not influence divorce rates. Empirical work contradicts this prediction. Friedberg (1998) demonstrates that divorce rates increased in the United States when changes in legal rules made unilateral divorce possible. ${ }^{3}$ My approach, however, makes a prediction that is consistent with the evidence, because it demonstrates that the frequency that relationships dissolve will increase if costs associated with identifying noncooperative behavior within marriage decline.

Formal enforcement operates in two ways to improve outcomes. It has the direct effect of reducing the immediate benefit of opportunistic behavior. Effective contract enforcement means (in this article) that an individual will, with positive probability, be required to repay gains obtained from violating a contract. The better the enforcement, the less attractive it is to violate the contract. Moreover, the mere fact that enforcement is costly creates an asymmetry between established and potential partnerships that provides an additional incentive for cooperation. If a partnership dissolves following opportunistic behavior and new partnerships begin only after parties make a costly investment in contracting, then players will resist the temptation to cheat in existing relationships in order to avoid paying the cost needed to start a new relationship.

Section III makes this point in a simple setting where there are no bargaining frictions: if a relationship ends, players can find a new partner immediately. When there is a moderately effective legal system, cooperation is possible. Lower levels of cooperation are possible without a legal system but only when partners find ways to impose costs on each other in the early stages of a relationship. The results in Section III show that this can be done by direct destruction of goods at the beginning of a relationship or by delaying the start of a productive relationship.

${ }^{2}$ This result appears in the work of Hosios (1994) and Mortensen and Pissarides (1994).

${ }^{3}$ Binner and Dnes (2001) provide similar evidence for England and Wales. 
In Section IV, I add a particular form of matching friction to the model. The probability of finding a new partner is equal to the fraction of unmatched agents, so that it is easier to find a new partner the more unmatched people there are in the population. Matching costs arise endogenously in this version of the model. Cooperative long-term relationships exist because if all relationships persist, there will be no unmatched partners available and therefore cheating on a relationship is extremely costly. It is still the case that formal means of contract enforcement could lead to shorter and more productive partnerships, although, curiously, some inefficient partnerships must persist when there is a low probability of a relationship going stale. This result is again a consequence of bargaining frictions: if relationships are unlikely to go stale, then in an equilibrium in which agents cooperate in productive relationships it will always be difficult to find a new partner.

Section II introduces the formal model. Following the main analysis of Sections III and IV, Section V reflects on who gains from the legal system and discusses the impact of a transition from relational contracting to contracting governed by enforceable contracting. Section VI discusses related literature. Section VII contains a discussion of variations of the model. Proofs of the propositions appear in this article's appendix.

\section{The Model}

Matching. Players are originally randomly matched to play a twoplayer game. Matched players are called partners and are said to be in a partnership or relationship. Partnerships last provided that both players agree to participate. The population of players is large. I neglect the possibility that players match with someone they have previously met.

Basic interaction. The payoff matrix below describes the prisoner's dilemma game played in the initial stage of a partnership. Assume that $b>a>0, d>0$ and that $2 a>b-d$. The last inequality guarantees that the outcome where both players cooperate yields the highest joint payoffs in the stage game.

\begin{tabular}{|l|c|c|}
\hline & Cooperate & Cheat \\
\hline Cooperate & $\mathrm{a}, \mathrm{a}$ & $-\mathrm{d}, \mathrm{b}$ \\
\hline Cheat & $\mathrm{b},-\mathrm{d}$ & 0,0 \\
\hline
\end{tabular}

Transition. Partnerships can be either fresh or stale. Fresh partnerships have stage-game payoffs described by the game described in the chart above. Stale matches provide a payoff $l, l \in(0, a)$, to both players as long as the partnership continues. New matches play the game in the chart, but the quality of a partnership may change after each period. With 
probability $\rho \in(0,1)$ a fresh match remains fresh. With probability $1-$ $\rho$, a fresh match becomes stale. Once a match becomes stale, it continues to be stale as long as the partnership continues. The probability $\rho$ is independent across match and across time period.

Information. Players know their own past actions, the past actions of their partners, and the quality of their current relationship (if any). Players cannot observe or learn the past actions of agents they have not met, nor can they learn the actions that their current partner took in previous relationships.

Enforcement. When enforcement is possible, agents simultaneously decide whether to invest in making a legal contract when they first meet. They then learn the amount invested by the other agent and play the stage game shown in the chart. The contract lasts for the life of the relationship. The quality of the legal protection offered by the contract depends on the quality of the legal system and the detail of the contract. The minimum investment determines contract detail. If $c$ is the minimum invested, then the legal system identifies cheating when it occurs with probability $Q(c)$, where $Q(\cdot)$ is increasing and $Q(0)=0$. The system never makes the error of identifying cheating when it did not occur. If cheating is identified, both players receive a payoff of zero. Contracts only provide an opportunity to rectify the damage done by cheating in one-shot interactions. They cannot be used to require long-term relationships or issue penalties. ${ }^{4}$ This form of restitution damage is a natural starting point for analysis. The informational assumptions imply that an agent knows only about performance in contracts he has signed. The population does not learn whether other agents have signed and honored or breached contracts.

Strategies. In each period of a fresh relationship, a player's strategy specifies an action in the game described in the chart followed by a decision to break the partnership. These choices depend on history (all past decisions of the player and his opponent). The decision to end a relationship comes after stage-game actions have been revealed. In all of my propositions, I limit attention to strategies that are stationary, which here means that a player's strategy depends only on the history of the play with his current partner. ${ }^{5}$

Payoffs. Players maximize the discounted sum of their stage-game payoffs, net of contracting costs. Players have a common discount factor, $\delta$, with $\delta \in(0,1)$.

Equilibrium. This article concentrates on stationary, subgame-perfect

${ }^{4}$ The legal system is not able to enforce punishments. If all other aspects of the model remain unchanged, the ability to enforce punishments against cheaters will make it is easier to support cooperation.

${ }^{5}$ The same qualitative results are available if I assume instead that players leave the population at a constant rate only to be replaced by new players and that the number of periods that a player has been in the population is not observable. 
equilibrium outcomes. I assume throughout that an agent will discontinue a partnership only if doing so is at least as attractive as continuing the partnership assuming that the opponent is willing to continue. ${ }^{6}$ Like most dynamic games, there are many equilibrium outcomes. I concentrate on equilibria with additional efficiency properties.

\section{No Matching Frictions}

This section illustrates the way in which formal enforcement mechanisms can change the nature of relationships. I maintain the simplifying assumption that unmatched agents can find a new partner without cost and future partners have no knowledge of an individual's past performance. I study the implications of matching frictions in the next section. The first subsection studies the model without enforcement, and the second subsection studies the implications of enforcement. The ability to enforce contracts improves performance for two reasons. It has the direct effect of reducing the gains from cheating on a contract. Somewhat more subtly, the cost of writing a contract makes it more profitable to remain in a relationship rather than start a new one.

\section{A. No Enforcement}

When there are no bargaining frictions and no way to observe and punish noncooperative behavior, agents are strongly tempted to cheat on their partners and move on to new relationships. If everyone is opportunistic in this way, however, there will be no cooperation. The following result is a simple consequence of this idea.

Proposition 1. Assume that there is no formal legal system and that matching is frictionless. There is no stationary, pure-strategy equilibrium outcome in which agents cooperate in their first interaction with a new partner.

Proposition 1 provides conditions under which there is no cooperation in new relationships. Plainly, if partnerships break up whenever one party cheats, there will be no cooperation at all. Some cooperation is possible in equilibrium when the strong restrictions on strategies assumed in proposition 1 do not hold. I suggest two possibilities in this section assuming that third-party enforcement is not possible. The common feature of these constructions is that cooperation is possible if it is costly to start new relationships. This theme appears later in this article. In Section III.B cooperation arises in part because agents must pay an enforcement cost to begin a relationship. In Section IV, matching frictions enable cooperation.

${ }^{6}$ Otherwise, partnerships can stop at any stage, since if the strategies of both players specify ending a partnership neither player can cause the relationship to continue through a unilateral action. 
Cooperation is possible if we relax the assumption that strategies do not depend on the time period. If agents condition their behavior on the time period, strategies could specify cooperation only in the first period but in no other new relationship. In this case, the continuation value of any unmatched agent is zero, so the threat of discontinuing a partnership is potent. The value of remaining in a cooperative relationship forever (taking into account that a relationship grows stale-and thereafter produces a future discounted payoff of $\bar{l}=l /(1-\delta)$-with probability $1-\rho)$, is

$$
V_{P}=\frac{a+\delta(1-\rho) \bar{l}}{1-\delta \rho},
$$

where $V_{P}$ is the value of a persistent partnership, in which agents stay together forever, cooperating while the relationship is fresh. It follows that cooperation is more attractive than cheating whenever $b \leq V_{P}$. Players will therefore be able to form (and maintain) cooperative partnerships if they can coordinate on an equilibrium using time-contingent strategies. The strategies that support this equilibrium are implausible because they rely on making a sharp distinction between the first period and future periods. In variations of the model in which old agents depart and new ones appear, these distinctions may not be feasible. There are, however, other ways to generate cooperative partnerships without formal enforcement.

For example, cooperation is also possible if agents are willing to stay in a relationship even after one or more periods in which neither player cooperated. Assume that players cheat for the first $N$ periods of a relationship and then begin cooperating. If $V_{n}$ denotes the value of a partnership that has interacted for $n$ periods but has not gone stale, then $V_{N}=V_{P}$ and, for $n<N$,

$$
V_{n}=\delta\left(\rho V_{n+1}+(1-\rho) \bar{l}\right) .
$$

Equation (2) reflects the possibility that until the partners start cooperating, there is no immediate value to the relationship and in the next period the value of the relationship is $\bar{l}$ if it goes stale and (by definition) $V_{n+1}$ otherwise. It follows that

$$
V_{0}=\frac{a(\rho \delta)^{N}+\delta(1-\rho) \bar{l}}{1-\delta \rho} .
$$

When players are patient and partnerships are unlikely to grow stale, it is possible to sustain an equilibrium in which agents stick with their partner but do not cooperate for the first few periods of the relationship. In the early periods of a partnership, equilibrium specifies that both agents cheat, so there is no short-term gain from deviating. Agents would not gain by leaving an established partnership because it will take even more 
periods to obtain a good payoff. Agents will not cheat in an existing relationship (i.e., one that is at least $N$ periods old and is not stale) provided that

$$
b-a \leq \delta\left(V_{N}-V_{0}\right)=\frac{\delta a\left(1-(\rho \delta)^{N}\right)}{1-\delta \rho} .
$$

Inequality (4) will hold for sufficiently large $N$ and $\delta$ and $\rho$ close to one.

This construction assumes that partnerships will last forever. If the payoff to stale partnerships is sufficiently low, it makes sense to break off these relationships. A similar construction allows cooperation in these situations as well.?

\section{B. Enforcement}

Proposition 1 suggests a role for enforcement procedures. Add a stage to the game in which agents simultaneously decide whether to write a legal contract. These investments are public. The quality of the legal protection offered by the contract depends on the quality of the legal system and the detail of the contract. The minimum investment determines contract detail. If $c$ is the minimum invested, then the quality of the contract will be $Q(c)$. If the quality of the contract is $Q(c)=q$, then the legal system identifies cheating when it occurs with probability $q$. If cheating is identified, both players receive payoff of zero.

Proposition 2 describes how the existence of a low-cost enforcement system increases the scope for cooperation. It identifies three qualitatively different equilibrium outcomes, depending on the effectiveness of the enforcement technology. Efficient partnerships are sustainable in equilibrium provided agents do not wish to cheat in a fresh relationship and they prefer to be in a new relationship rather than extend a stale one. This is possible if it is inexpensive to enforce agreements that punish cheaters. Increasing enforcement costs raises the set-up costs associated with forming new partnerships. If these costs are sufficiently high, agents will prefer to remain in stale relationships rather than to start new ones. Further increases in enforcement costs lead to a return of the conclusion of proposition 1: cooperation is not possible in a symmetric, stationary equilibrium.

In order to state the proposition formally, I introduce some terminology. Let $\Delta=(a-l) /(1-\rho \delta)$, where $\Delta$ describes the additional value of being in a series of productive partnerships (in which agents cooperate while the match is fresh and terminate the relationship as soon as it

\footnotetext{
${ }^{7}$ While Lindsey, Polak, and Zeckhauser (2001) focus on other conventions for supporting cooperation, they point out that having relationships begin with a period of abstinence is a convention that leads to (nearly) efficient equilibrium outcomes in their model.
} 
becomes stale) over being in a persistent partnership. Plainly, $V_{P}>\Delta$. Let $c_{P}$ be defined to satisfy

$$
b\left(1-Q\left(c_{P}\right)\right)=a+\delta \rho c_{P} .
$$

Equation (5) has a unique solution. Similarly, let $c_{E}$ be defined to satisfy

$$
b\left(1-Q\left(c_{E}\right)\right)=V_{P}+\delta c_{E},
$$

where $c_{P}$ and $c_{E}$ represent the minimum expenditure in legal costs needed to guarantee cooperation in, respectively, persistent partnerships and productive partnerships.

Let $c^{*}=\min \left\{c_{P}, c_{E}\right\}$. One can check that $c_{P}=c_{E}$ when $c_{P}=\Delta$ and

$$
c^{*}= \begin{cases}c_{p} & \text { if } c_{p} \leq \triangle . \\ c_{E} & \text { if } c_{p} \geq \triangle .\end{cases}
$$

Proposition 2. Assume that there is a formal enforcement mechanism. If $c^{*} \leq \Delta$, then there exists an equilibrium with productive partnerships. If $c^{*} \in\left[\Delta, V_{P}\right]$, then there exists an equilibrium with persistent partnerships. If $c^{*}>V_{P}$, then joint cooperation is not sustainable in the initial stage of a symmetric, stationary, subgame-perfect equilibrium.

Here $c^{*}$ is a measure of the quality of the formal legal system. To better understand this measure, examine equation (5). The left-hand side of the equation is what an agent expects to gain from cheating in a productive relationship assuming that his partner cooperates and each agent contributes $c_{E}$ to enforcement. The right-hand side describes the payoff to cooperation if agents stay in relationships as long as they are fresh. There is an immediate payoff of $a$. Furthermore, if the agent cooperates and the relationship remains fresh, then the agent does not need to pay contracting costs in the next period. This leads to an expected savings of $\delta \rho c^{*}$. Consequently, $c_{E}$ is the minimum expenditure on enforcement needed to deter cheating if relationships last as long as they are fresh. Similarly (although somewhat less transparently), the right-hand side of equation (6) measures the gains from cooperating in the cooperative phase of a relationship that will persist even when stale; ${ }^{8} c^{*}$ is the minimum expenditure needed to sustain any cooperation.

The intervals of existence of the different equilibria respond intuitively to parameter shifts. Improvements in the legal system (shifts that increase the probability of detecting a cheat for any investment $c$ ) lower $c^{*}$ and hence make contracting more effective. Increases in $a, \delta$, or $\rho$ or decreases

${ }^{8}$ Here $V_{P}$ is equal to payoff from a persistent relationship. Cheating will force an agent to pay contracting costs of $c$ in the next period; these costs will not be paid if the agent stays in the relationship. Consequently, the second term on the left-hand side of (6) represents the savings in contracting costs associated with playing according to the equilibrium. 
in $b$ make cooperation more attractive. These shifts lower $c^{*}$ and raise both $\Delta$ and $V_{P}$, meaning that it requires less powerful formal legal systems to sustain cooperation.

Improved enforcement mechanisms lead to more contracts and more productive partnerships. Incremental improvements in the enforcement technology can be valuable. At first, the system enables individuals with high $\rho$ to form persistent, cooperative relationships. Once it is valuable for these partnerships to write contracts, subsequent improvements have two effects: they lower the cost of contracting to those relationships that are already using formal enforcement, and they expand the set of partnerships that use enforcement. When enforcement is sufficiently cheap, there will be three kinds of relationships: those with high $\rho$ terminate relationships as soon as they become stale, those with intermediate $\rho$ remain in persistent relationships, and those with low $\rho$ cannot take advantage of enforcement.

Improvement in the enforcement technology may lead to either an increase or a decrease in the total expenditure on contracts. For most $\rho$ an improvement in the enforcement technology reduces the amount spent on contracting without changing the qualitative form of the contracts. For marginal types, however, an improvement in technology will either make it valuable to support a persistent cooperative relationship with a contract or lead to a switch from writing a single contract to support a persistent relationship to writing a series of contracts to support fresh relationships. These discrete increases in expenditures may well exceed the reductions caused by the improvement in technology. ${ }^{9}$

\section{Matching Frictions}

This section introduces a friction into the matching process. The friction makes it costly to find a new partner when a relationship ends and therefore makes cooperation easier to sustain. I assume that the probability of finding a new partner is proportional to the number of unmatched agents. Consequently, if most agents are in relationships, then it is difficult to find a new partner. When it is difficult to find a new partner, the relative value of having a partner increases. Individuals may be more willing to remain in existing relationships because they know that it is costly to initiate a new relationship.

Specifically, I search for steady-state distributions of partnerships. Such a distribution specifies the fraction of unmatched agents, $w$, the fraction

${ }^{9}$ The cost of a contract needs to be paid only once during the lifetime of a partnership. A small shift in contracting costs can lead to a shift from persistent relationships to productive ones. This change, in turn, creates a large increase in the number of times an individual contracts and a corresponding increase in the expenditure on contracts. 
of agents in fresh relationships, $r$, and the fraction of agents in stale relationships, $s$. To describe the steady-state conditions generally, assume that a fraction $\tau, \tau \in[0,1]$, of stale relationships continue. In a steady state, it must be the case that the fraction of each type of relationship that forms in a period is balanced by the fraction that breaks up. Consequently, $r, s$, and $w$ must satisfy $r+s+w=1$, and

$$
\begin{gathered}
(1-\rho) r=\rho w^{2}, \\
(1-\tau) s=(1-\rho) \tau\left(r+w^{2}\right),
\end{gathered}
$$

and

$$
w^{2}=(1-\tau)\left[(1-\rho)\left(r+w^{2}\right)+s\right] .
$$

The left-hand side of equation (7) is the fraction of fresh partnerships that become stale in any period. The right-hand side is the fraction of new fresh matches formed. The left-hand side of equation (8) is the number of stale partnerships that break up, which must be balanced in steady state by fresh partnerships that go stale but do not break up, while the right-hand side is the fraction of relationships that become stale but do not break up. Equation (9) has a similar interpretation.

This analysis modifies the results of the previous section in two ways. First, the expectation that people will stay in partnerships forever is an equilibrium (under mild assumptions). If everyone expects partnerships to last forever, then no one will breach a relationship because they know that they will never find another partner. Hence, even without formal enforcement mechanisms, long-term cooperative relationships are possible in equilibrium. Second, if there is a small probability that relationships go stale, then even with effective contract enforcement some stale relationships will persist in equilibrium. Otherwise, new partners would be so hard to find that players would be unwilling to end relationships.

In Section IV.A I show that matching frictions may make it possible to support cooperation in a stationary equilibrium even without enforcement. In order to support cooperation, stale partnerships persist. In Section IV.B I consider the possibility of an equilibrium in which only fresh partnerships exist. Even with the possibility of enforcement, these outcomes do not exist when $\rho$ is close to one and may not exist when $\rho$ is close to zero. Enforcement can improve performance for some parameter values.

\section{A. Persistent Partnerships}

The first case to consider is when agents always remain in low relationships. Here the punishment for breaking a relationship is severe- 
you never find another partner. Long-term relationships exist under weak conditions.

Proposition 3. Assume that $b<V_{P}$. There exists a steady-state equilibrium outcome in which agents always cooperate in equilibrium.

Recall from (1) that $V_{P}$ is the value of a relationship that lasts forever and in which agents cooperate while the relationship is fresh. Since $a>$ $l$, this quantity exceeds $l$, and so a simple sufficient condition for the existence of a persistent relationship with cooperation is $b \leq \bar{l}$, which holds if $\delta$ is sufficiently close to one.

Proposition 3 contrasts with the analysis of Section III.A. The existence of matching friction makes it possible to sustain cooperation without enforcement even in stationary equilibria. Proposition 3 limits attention to equilibria in which no agent leaves a relationship in equilibrium. In the next section, I discuss the existence of equilibria in which agents cooperate in fresh relationships but terminate at least a fraction of stale relationships.

\section{B. Productive Partnerships}

The previous subsection demonstrated that with matching frictions it is possible to support an equilibrium with cooperation but in steady state agents remain in relationships that no longer provide high payoffs. At the other extreme is the equilibrium in which people have productive partnerships in the sense that they cooperate and remain paired if and only if the partnership is fresh. This type of equilibrium has the potential to be superior to the persistent equilibrium for two reasons. First, more partnerships earn high payoffs. Second, when people break off relationships, there are unmatched agents. Therefore, it is easier to find partners. The second factor makes cheating more attractive and therefore enforcement more costly. In this subsection, I examine the existence of equilibria in which partnerships are productive. I include the possibility of formal enforcement in the analysis.

When there are matching frictions and the probability of a match becoming stale is sufficiently low, then there does not exist an equilibrium in which all partnerships are productive. This result is a simple consequence of the matching technology. Imagine a population in which all players cooperate in fresh relationships but break off a relationship if either partner cheats or a relationship becomes stale. When $\rho$ is close to one, few relationships break up. Consequently, an agent without a partner will find it difficult to get a new partner and will be better off remaining in a stale relationship.

It may also be difficult to sustain an equilibrium with productive partnerships when $\rho$ is small. When $\rho$ is close to zero, fresh partnerships do not last long. If agents remain in partnerships only when they are fresh, 
then it will not be hard to find new partners. Consequently, it is not costly for an agent to find a new partner. Unless enforcement is effective, it will be tempting for an agent to cheat on a partner and move on to a new relationship. The next proposition summarizes these observations.

Proposition 4. There exists $\bar{\rho} \in(0,1)$ such that if $\rho \in(\bar{\rho}, 1)$, there does not exist a productive steady-state equilibrium. If $a<b(1-Q(a-l))$, then there exists $\underline{\rho} \in(0,1)$ such that if $\rho \in(0, \underline{\rho})$, there does not exist a productive steady-state equilibrium.

The condition $a<b(1-Q(a-l))$ identifies what it means for enforcement costs to be sufficiently low. If agents agree to spend $c$ on enforcement and $a \geq b(1-Q(c))$, then cheating will be deterred. If $a<b(1-Q(a-l))$ holds, then the cost of deterring cheating must exceed $a-l$. On the other hand, if $\rho$ is close to zero, the average payoff (not including enforcement costs) of staying in fresh relationships is $a$ while the average payoff of a stale relationship is $l$. Since enforcement costs must be paid in nearly every period when $\rho$ is small, the average net value of being in a series of fresh relationships is roughly $a-c$, which must exceed $l$ to deter agents from remaining in stale relationships. So if it costs more than $a-l$ to prevent cheating and $\rho$ is close to zero, it is too expensive to maintain productive relationships.

Depending on the quality of formal enforcement, the economy has differential incentives to maintain the quality of partnerships. Compare the equilibria in two low $\rho$ economies, one in which enforcement is costly, the other in which it is cheap. In the first economy, proposition 4 implies that agents will maintain long-term relationships even after they have gone stale. In the second economy, enforcement supports the existence of a productive equilibrium. The first economy benefits greatly from effortseither economy wide or by bilateral pairs-to increase $\rho$. Such efforts have little benefit when enforcement is inexpensive and new partners are easy to find.

According to proposition 4, the potential for productive partnerships may break down when there are matching frictions. This leads to the somewhat surprising conclusion that formal enforcement may not benefit agents in the presence of matching frictions. To see this, assume that an equilibrium with productive partnerships does not exist (even with enforcement). Any equilibrium with cooperation must involve the survival of some stale partnerships. If, however, stale partnerships remain with positive probability, then all agents weakly prefer to remain in these partnerships rather than search for new ones. Hence, the expected equilibrium payoff can be no greater than $V_{P}$. Proposition 3 gives conditions under which this payoff can be sustained without enforcement. Corollary 1 summarizes this result.

Corollary 1. Assume $b<V_{P}$ and $a<b(1-Q(a-l))$. There exists 
$\rho, \bar{\rho} \in(0,1)$ such that if $\rho \in(0, \rho) \cup(\bar{\rho}, 1)$, then formal enforcement does not increase the maximum equilibrium total payoff.

Corollary 1 states that when there are bargaining frictions and partnerships are either very likely to remain fresh or very likely to go stale, then the ability to enforce contracts does not improve outcomes. The conditions in proposition 1 are precisely the ones used in propositions 3 and 4 . The values $\underline{\rho}$ and $\bar{\rho}$ can be taken to be the same as the values in proposition 4.

Corollary 1 does not mean that enforcement has no role when there are matching frictions. Specifically, if $b \geq V_{P}$, then it is not possible to guarantee cooperation without enforcement. ${ }^{10}$ The proof of proposition 4 in the appendix characterizes equilibria in which there is a positive fraction of stale partnerships in steady-state equilibrium.

The stark claim that enforcement does not improve performance is a residual of modeling assumptions. The proposition depends strongly on the assumption that stale relationships are not strategic. If enforcement is needed to guarantee a payoff of $l$ in a stale relationship, then it will generally be beneficial when there are frictions. Alternatively, if the value of safe relationships varies, then when $\rho$ and $\delta$ are sufficiently close to one, there exists an equilibrium with enforcement in which a fraction of the stale relationships-the least profitable ones-end, creating a sufficiently large pool for finding new matches. In such a model, effective enforcement serves to shorten the length of partnerships and to increase a partnership's average payoff, just as in the previous section.

Even when the conditions of proposition 4 hold, partnerships with productive equilibria may exist for some intermediate values of $\rho$. To see this, consider the case when no enforcement is possible. In order to have a productive equilibrium, two conditions must be satisfied. First, it must be in the best interest of partnerships not to cheat when they are in fresh relationships. Second, it must be more profitable to break off a stale relationship and search for a new partner than to continue. I have explained that the first condition is impossible to satisfy (without inexpensive enforcement) if $\rho$ is close to zero and the second condition is impossible to satisfy when $\rho$ is close to one. The next result states the conditions needed for a productive steadystate equilibrium without enforcement. In the proposition, $w^{*}(\rho)=\left\{-(1-\rho)+\left[(1-\rho)^{2}+4 \rho(1-\rho)\right]^{-1 / 2}\right\} / 2 \rho$ is the steadystate fraction of unmatched agents when there are no stale partnerships.

${ }^{10}$ Enforcement also increases payoffs off the equilibrium path: the value of being without a match will be higher in an equilibrium with enforcement and a nontrivial break-up probability of stale partnerships than in the persistent equilibrium in which no partnerships break up. 
That is, $w^{*}(\rho)$ is the solution to the system of equations (7), (8), (9), and $r+s+w=1$ with $\tau=0$.

Proposition 5. If

1. $a / b \geq 1-\delta \rho\left(1-w^{*}(\rho)\right)$ and

2. $\quad a / l \geq\left[1-\delta \rho\left(1-w^{*}(\rho)\right)\right] / w^{*}(\rho)$,

then there exists a productive steady-state equilibrium.

Under the conditions in proposition 5 , there exists an equilibrium in which agents always cooperate and never remain in a stale relationship, even without formal enforcement. The first condition guarantees that an agent does not want to cheat when he is in a fresh relationship (and his partner cooperates). The left-hand side of the inequality is less than one. The right-hand side is decreasing in $\rho$, equal to 1 when $\rho=0$, and equal to $1-\delta$ when $\rho=1$. Therefore, it cannot be satisfied when $\rho$ is small and can be satisfied for large $\rho$ when $\delta$ is sufficiently close to one. The second condition in proposition 5 guarantees that agents want to leave stale relationships. The left-hand side is greater than one. The right-hand side is equal to one when $\rho=0$ and is arbitrarily large for $\rho$ close to one $\left(w^{*}(1)=0\right)$. It is plainly possible to satisfy both conditions for some $\rho$ if $\delta$ and $a / b$ are close to one.

Proposition 5 provides sufficient conditions for the existence of a productive steady-state equilibrium. The ability to enforce contracts strictly enlarges the set of $\rho$ for which this type of equilibrium exists by lowering the benefits from cheating (i.e., by relaxing the first condition in proposition 5).

\section{An Alternate Form of Friction}

This subsection notes that a simple kind of friction has little effect on the results of Section III. If $\lambda$ represents the probability of finding a match (independent of what others are doing), then one can think of Section III as an analysis of the case $\lambda=1$. Let $V$ denote the value to someone in an established fresh partnership, and let $W$ denote the value of an unmatched agent. It follows that

$$
W=\lambda(V-c)+(1-\lambda) \delta W .
$$

In general, we can repeat the computations from the proof of proposition 2 to generalize earlier results. The details are tedious and not instructive, so I just briefly summarize the result.

Increasing frictions (decreasing $\lambda$ ) reduces both $W$ and $V$, with $W$ decreasing more than $V$ since unmatched agents are certain to incur matching costs. In order to rule out opportunistic behavior, it is necessary that

$$
V-\delta W \geq b(1-Q(c)) .
$$

The left-hand side of this expression may go up or down when matching 
frictions increase. However, when $\delta$ is close to one, frictions substitute for enforcement: inequality (10) will hold for smaller values of $c$. Consequently, when $\delta$ is close to one, including frictions that delay matching at a rate that is independent of the fraction of established partnerships in the population makes it possible to support cooperation at lower enforcement cost. Otherwise, the qualitative conclusions of Section III continue to hold.

\section{Improving Enforcement}

The existence of effective means to deter cheating changes the nature of partnerships and has the potential to improve outcomes. This section considers ways to make enforcement better. Since relational contracting is able to sustain some cooperation, it is useful to consider how an economy may move toward improved enforcement. Particular studies suggest that the transition process is not unique. Greif (1994) observes that the organization of society led to a development of formal enforcement in the individualistic Genoese society but not among the collectivist Maghribi traders. Allen et al. (2005) argue that China's vast size as well as its cultural traditions make dramatic changes in institutions far less likely than in other transition economies. Certainly for the case of China, it is worthwhile to investigate whether there are advantages to incremental improvements in enforcement.

Most of the discussion is informal. The limited technical claims are straightforward consequences of the earlier analysis, so I do not provide detailed proof.

There are at least two ways in which there could be public provision of enforcement. First, a central authority can improve the verification technology so that a fixed expenditure on the contract leads to a higher probability that cheating will be detected. Formally, one would model this as a shift in the monitoring function $Q(c)$. If everyone in the population had access to this technology, then improvements in enforcement would have external benefits. Individuals would have little (or, in the idealized large population of the model, no) incentive to make costly investments to improve the technology. Second, one could imagine subsidies that reduce the cost an agent would pay for a particular level of enforcement without improving the technology itself. The equilibria constructed in Section IV create the proper incentives for investment. Hence, subsidizing access to the enforcement technology, for example, by providing a rebate on a fraction of the contracting costs to both agents, would lead to a level of contracts that is greater than what is socially optimal. This follows for two reasons. Agents would no longer need to pay a contracting cost to start a relationship. This makes it less expensive to break up a relationship, and therefore one must pay more to deter cheat- 
ing. Also, if the costs of contracting are not paid directly, then agents will have no incentive to remain in stale relationships. Consequently, contract length may be too short given that enforcement is costly. These remarks suggest that without bargaining frictions, publicly provided law leads to overinvestment in contracting.

In the basic model, no one loses from the existence of the enforcement mechanism, but individuals who have access to more stable relationships gain more, so one would expect these groups to provide the support needed to create a system. Incremental efforts to create effective enforcement where there previously had been none are strictly beneficial in the short run to only those individuals with access to high $\rho$ partnerships. On the other hand, there are potentially large benefits to be made from even small improvements in enforcement. As the system improves, more and more individuals gain from further improvements. Without specifying the cost of making improvements, it is not possible to characterize a socially optimal $Q(\cdot)$ function, but it is at least plausible to assume that one could obtain this level through a series of incremental improvements.

\section{Related Literature}

Lindsey et al. (2001) present an insightful analysis of a related model in which there is pairwise matching and the value of the stage game depends on independent, identically distributed private information that does not persist. There are no bargaining frictions. In each period, a player must decide whether to transact with his partner and then whether to continue the relationship or to deal with someone else. Transactions may be socially beneficial but privately costly (if one agent loses a little while the other gains a lot). The authors show that any strongly symmetric equilibrium outcome that maximizes equilibrium payoffs can be obtained if players follow one of two intuitive behavioral rules (conventions). One convention dictates that a partnership ends when either player fails to transact. The other convention dictates that a partnership ends when one player fails to transact.

My model has symmetric information throughout, so agents will have identical incentives to break off relationships and my analysis focuses on the convention in which a relationship breaks up following a unilateral failure to cooperate. In constructing symmetric equilibria, continuations following cheating by both agents can be arbitrary. So, conventions that only terminate a relationship following joint deviations play no essential role in my analysis. Lindsey et al. (2001) observe that the value of a partnership increases over time and, in long-lived relationships, partners transact more than is socially optimal. Inefficiency arises because agents are willing to take short-term losses in order to preserve a mature rela- 
tionship (rather than run the risk of causing an end to the relationship and initiating a new, less valuable, partnership).

Kranton (1996) and Carmichael and MacLeod (1997) both make the observation that imposing costs at the beginning of a relationship can lead to efficiency gains. Both papers primarily focus on complete-information models in which agents play two-player stage games with a prisoner's dilemma character. ${ }^{11}$ Following each stage, players can continue or find a new partner. Carmichael and MacLeod (1997) demonstrate that strategies involving gift exchange at the beginning of a relationship are evolutionarily stable. Gifts in their model play precisely the same role as costs in my model. In Kranton's (1996) model, high payoff equilibria involve increasing cooperation over time. Section III of this article echoes their results. ${ }^{12}$

Ramey and Watson (1997) study a model in which individuals play prisoner's dilemma type games in a changing environment. Partners must make specific investments. High investments lead to partnerships that persist through good and bad economic conditions. Low investments lead to fragile partnerships that break up in bad states. Ramey and Watson show that when the low productivity state is sufficiently unlikely, partners do not make investment decisions, choosing instead to discontinue relationships in bad states. An effective enforcement mechanism makes it possible to construct qualitatively similar equilibria in my model. Contracting cost plays the role of relationship-specific investment. When there is a small probability that a relationship will become stale, then relationships in my model also tend to be fragile.

McMillan and Woodruff (2000b) and Dixit (2003) are two of many discussions that emphasize how availability of formal legal institutions changes the way in which information about agents spreads through the economy. When there are reliable ways to enforce contracts, agents do not need to know much about their trading partners. When enforcement is not reliable, it is more important for individuals to collect information about current and potential partners. Information can be distributed through middlemen, trade associations, or informal networks. There is a role for these mechanisms of providing information even in settings where, as in my model, stage-game payoffs are common knowledge. Several articles have illustrated how the ability to condition on information about past performance can improve the ability to cooperate. In my basic model, one could imagine that players learn, either by paying a third party, by

${ }^{11}$ Kranton (1996) also discusses an incomplete information variation.

${ }^{12}$ Sobel (1985) and Watson (1999) present models in which the scale of operations increases over time in a (potentially) long-term relationship. In these models, by varying the scale of interaction that is an equilibrium, players learn about a characteristic of their partner. 
trading within a network, or through word of mouth, the history of a potential opponent's play. Players could then use this information to determine with whom they will cooperate. This approach appears in the work of Milgrom, North, and Weingast (1990) and Kandori (1992), among others.

In Dixit (2003), different individuals have different propensities to cooperate. Access to information about past behavior permits people to draw inferences about the attractiveness of potential partners. He reviews ways in which information can flow across trading partners. McMillan and Woodruff $(1999,2000$ a) analyze survey evidence about how much energy people devote to investigating potential trading partners (and their unwillingness to go with new partners simply because they get better offers). McMillan and Woodruff $(1999,2000 \mathrm{~b})$ emphasize that there is a substitution between formal and informal enforcement of contracts. They point out that search costs may improve the performance of relational contracting by giving trading partners incentives to maintain current relationships rather than look for new partners. The results in Section IV are consistent with their finding.

McMillan and Woodruff (2000a) find that partners go to great lengths to preserve relationships. Vietnamese businessmen avoid the strong punishment of breaking off dealings after a partner fails to provide a prompt payment. McMillan and Woodruff (1999) suggest that this behavior is the result of incomplete information. Firms must try to decide whether a poor outcome resulted because the partner cheated or is intrinsically unreliable or because of a short-term shock that reduced the partner's ability to cooperate. In practice, it is in the interest of firms to develop a reputation for forgiving their partners. In my model, there is no scope for this consideration. In prominent cases, the highest payoff equilibria demand that players breach relationships after someone cheats. Efforts to maintain relationships in spite of poor short-term performance would certainly arise in models with private information.

\section{Conclusion}

This article studies different ways to obtain cooperative long-term partnerships. Long-term relationships can substitute for enforcement mechanisms but at the cost of limiting mobility. In the model of this article, agents will stay trapped in stale relationships rather than begin new ones that have greater gains from trade. This section reviews the importance of some of my assumptions.

I have made a few modeling assumptions that, while apparently restrictive, do not reduce the scope of the analysis. The analysis assumes that the shocks to a relationship take a simple form. Stale relationships never become productive again. This assumption simplifies notation but 
does not substantively affect the analysis. The important feature in the model is that there is a positive probability that the value of a relationship will decrease. Even if stale relationships sometimes become fresh again, the critical factor is how to behave when new partners provide superior trading opportunities than existing partners can provide. The simple message of this article is that in the absence of effective enforcement procedures, there will be a greater tendency to continue relationships even when more profitable relationships exist.

I have assumed that the probability that a relationship goes stale is independent across relationships. If, instead, the probability of going stale is perfectly correlated, then the matching frictions described in Section IV are not a problem-everyone can quit a relationship when it goes stale under the assumption that everyone else is quitting at the same time.

The model assumes that bargainers pay contracting costs at the beginning of a relationship. The important feature for this article's qualitative results is that the first contract is more expensive than later ones.

There are more significant limitations to my modeling approach. The analysis assumes that trading partners have symmetric information. This formulation limits the extent to which enforcement mechanisms work by gathering and validating information. Knowledge of an individual's history does not, in my model, provide information about that individual's preferences. Dixit (2003) assumes that different agents have different preferences. Including private information would create the possibility that agents cheat with positive probability even when they have signed contracts. If there is asymmetric information about whether cheating will be detected or differential incentives to cheat, it may be inefficient to write contracts that deter all cheating. Furthermore, if there are reasons why good partners may temporarily fail to cooperate, efforts to maintain relationships after apparent cheating (as documented by McMillan and Woodruff [2000a]) would arise.

It would be useful to permit enforcement agencies to publicize their findings. In my analysis, only parties to a transaction know whether a contract has been honored. Trade associations, informal networks, and public enforcement all have the potential to provide information about trading histories. How far this information is spread, how credible it is, and how it is used in equilibrium have important implications for the value of enforcement procedures.

A more detailed model of information flows also might enable a comparison of different types of enforcement. The "enforcement" that I discuss may be provided by a legal system sponsored by the government. It may as well be provided by any third party with access to information and mild sanctioning authority. Private enforcement is possible but, arguably, may rely on authority valid only within isolated communities (placing limits on the range of potential partners) or may lead to extreme 
punishments. A more detailed model would also allow one to investigate the implications of modifying the punishments for violating the contract.

I do not permit investments that improve the productivity of the relationship. Ramey and Watson's (1997) model shows how this can be done. Investments in contracting have some characteristics of relationshipspecific investment. These investments influence the cost of beginning new partnerships and persistence of relationships in ways captured by my model. On the other hand, building up the scale of a relationship may also increase opportunities for cheating. In models with the opportunity for ongoing relationship-specific investment, there may be a role for contracting in mature relationships. I have in mind situations where partnerships start small, either to impose costs of starting new relationships or to gather information, and then increase in scale. In these situations the potential gains from opportunistic behavior (and the need for enforcement) may arise only after investments have been made.

\section{Appendix}

\section{Proofs of Propositions 1-5}

Proof of proposition 1. Fix an equilibrium, and assume that players in new relationships cooperate. $b /(1-\delta)$ is the most that the agent can get in any play of the game, since $b$ is the largest stage-game payoff. By stationarity, when matching is frictionless and every agent cooperates in his first interaction with a new partner, a deviating agent can achieve $b /(1-\delta)$ by cheating and entering the pool of unmatched agents. Further, if the agent cooperated when he met a new partner, then his payoff would be strictly less. It follows that cooperation with probability one cannot be an equilibrium strategy. QED

Proof of proposition 2. Assume that agents adopt strategies that lead to productive partnerships. They invest $c^{*}$ at the start of a new relationship and cooperate in the relationship as long as the relationship remains profitable and their partner cooperates. When the relationship goes stale (or if anyone cheats), agents start a new relationship. Let $W$ denote the payoff of an unmatched player, and let $V$ denote the payoff of a matched player. For these strategies to be in equilibrium, two conditions must hold. First, it must be the case that agents do not wish to cheat (rather than cooperate) in a fresh relationship. Second, it must be the case that agents prefer to break off partnerships as soon as they become stale.

Consider the cheating condition. If an unmatched player meets a new player, contracts, and cooperates, then he obtains $-c^{*}+V$. If he cheats, then he gets $-c^{*}+b(1-q)+\delta W$. Therefore, a necessary condition for cooperation to be an equilibrium is that

$$
V \geq b\left(1-Q\left(c^{*}\right)\right)+\delta W .
$$


When agents cooperate, they earn an immediate payoff of $a$, followed by a continuation value of $V$ or $W$, depending on whether the relationship persists. It follows that $V=a+\delta \rho V+\delta(1-\rho) W$ and, since $W=-c^{*}+$ $V$,

$$
V=\frac{a-\delta(1-\rho) c^{*}}{1-\delta}
$$

and

$$
W=\frac{a-(1-\delta \rho) c^{*}}{1-\delta} .
$$

In order for this to satisfy inequality (A1) it must be that

$$
b\left(1-Q\left(c^{*}\right)\right) \leq V-\delta W=a+\delta \rho c^{*} .
$$

Inequality (A4) holds since $c^{*} \geq c_{P}$.

It follows from the definition of $\Delta$ and equation (A3) that $\bar{l} \leq W$ when $c^{*} \leq \Delta$. Hence, if $c^{*} \leq \Delta$, then an agent prefers to discontinue stale relationships rather than persist.

When $c^{*}>\Delta$, agents do better in persistent relationships than in productive ones. Assume that players cooperate in productive games and continue in partnerships for as long as there is no cheating (whether or not the relationship grows stale). Valuations are

$$
V=\frac{a+\delta(1-\rho) \bar{l}}{1-\delta \rho}
$$

and

$$
W=V-c^{*}
$$

Individuals will not cheat provided that

$$
b\left(1-Q\left(c^{*}\right)\right) \leq V-\delta W=\frac{(1-\delta) a+\delta(1-\rho) l}{1-\delta \rho}+\delta c^{*} .
$$

Inequality (A7) will hold whenever $c^{*} \geq c_{E}$. Moreover, the players will do better investing $c^{*}$ in a contract that supports a persistent relationship than staying out of relationships (or, equivalently, paying nothing to contract and then cheating) provided that $W \geq 0$, which implies that persistent partnerships are in equilibrium when $c^{*} \in\left[\Delta, V_{P}\right]$. When $c^{*}>V_{P}$, cooperation is not possible in equilibrium. Players must pay at least $c^{*}$ to enforce cooperation in partnerships, but if they pay that much, then the value of writing a contract that leads to a cooperative relationship is negative. QED

Proof of proposition 3. Assume that players cooperate whenever they 
are in new relationships and whenever their current partner has cooperated in the past. If their partner cheats, then the relationship ends. Under these conditions, the value of being in a fresh match is $V_{P}$ and the value of being unmatched is $W=0$ because the steady-state probability of being unmatched $w$ is equal to zero if agents use their equilibrium strategies. Since cheating is not attractive if $b=b+\delta W \leq V_{P}$, the proposition follows. QED

Proof of proposition 4. I begin by characterizing conditions under which a productive steady-state equilibrium exists. Assume that agents cooperate on the equilibrium path but break off a relationship as soon as it is no longer possible to obtain the high payoff $a$. Under these assumptions, the value of good relationship is given by

$$
V=a+\delta \rho V+\delta(1-\rho) W,
$$

where equation (A8) reflects the fact that good relationships degenerate with probability $1-\rho$.

The value for an unmatched agent is

$$
W=w(-c(q)+V)+(1-w) \delta W .
$$

With probability $w$, the agent finds a new partner. These agents decide how much to invest in contracting and (on the equilibrium path) begin a relationship that has value $V$. With probability $1-w$, the agent does not find a partner. Equation (A9) reflects the assumption that an agent earns zero while unmatched and then has a continuation payoff equal to $W$.

Finding the value functions involves solving two linear equations (A8) and (A9) for $V$ and $W$ :

$$
V=(1-\delta)^{-1}(1-\delta \rho(1-w))^{-1}\{a(1-\delta(1-w))-\delta(1-\rho) w c\}
$$

and

$$
W=(1-\delta)^{-1}(1-\delta \rho(1-w))^{-1} w\{a-(1-\delta \rho) c\} .
$$

Contracting cost must be sufficient to deter cheating. Hence, $c$ must satisfy

$$
b(1-Q(c))+\delta W \leq V .
$$

Let $r$ be the steady-state probability that an agent is in a high-level partnership and $w$ be the probability of being unmatched. It follows that $r+w=1$, because agents never stay in low-level partnerships. Furthermore, if $r$ is the fraction of high-level partnerships in one period, then in the subsequent period a fraction $\rho r$ of the population continue to be in their partnership, which the fraction $\rho$ of the $w^{2}$ new relationships remain highly productive. Consequently, the steady-state 
probabilities must satisfy

$$
r=\rho r+\rho w^{2}
$$

so, since $r=1-w$,

$$
w^{*}(\rho)=\frac{-(1-\rho)+\sqrt{(1-\rho)^{2}+4 \rho(1-\rho)}}{2 \rho} .
$$

The efficient level of expenditure on contracts, $c(\rho)$, solves inequality (A12) as an equation when $w=w^{*}(\rho)$ is defined by (A14) and equations (A10) and (A11) define $V$ and $W$. It is straightforward to show that equation (A14) has a unique solution for each $\rho$. The solution, $w^{*}(\rho)$ is a decreasing function of $\rho$.

The construction has provided value functions, a level of enforcement, and a steady-state distribution. In a productive equilibrium all agents pay $c$ at the start of a new relationship, cooperate as long as the relationship is fresh, and discontinue a relationship as soon as anyone cheats or the relationship becomes stale. Such an equilibrium exists if and only if $W$ is greater than or equal to $\bar{l}$, the value of a stale relationship, which from equation (A11) can be written

$$
\frac{w(a-(1-\delta \rho) c)}{1-\delta \rho(1-w)} \geq l .
$$

It follows from (A14) that $w^{*}(1)=0$. Therefore, inequality (A15) cannot hold when $\rho$ is sufficiently close to 1 . This proves the first part of proposition 4.

When $\rho=0$, (A15) simplifies to $c \leq a-l$, where (from [A12] solved as an equation) $c$ solves $a=b(1-Q(c))$. Hence, unless enforcement costs are low, at least some relationships will persist when $\rho$ is close to zero. This proves the second part of proposition 4.

It is possible to show that the left-hand side of inequality (A15) is increasing in $\rho$ at $\rho=0$, and one can construct examples in which there is an interval of $\rho$ values strictly between 0 and 1 that satisfy (A15).

When it is not possible to satisfy inequality (A15), it is possible to construct equilibria in which some stale relationships continue while others break up. If this is the case, then $V=V_{P}$ and $W$ is given by equation (A8) as before, but it also must be the case that $W=\bar{l}$ if agents are indifferent between breaking or maintaining low-value relationships. Using the expression for $W$, it follows that

$$
w=\frac{(1-\delta \rho) l}{a-\delta \rho l-c \delta(1-\delta \rho)} ;
$$

$w$ is also determined by the steady-state balance conditions. Let the frac- 
tion $\tau, \tau \in(0,1)$, be the probability that partners agree to continue in stale relationships. In steady state, the fractions of agents in high partnerships, low partnerships, and no partnership $(r, s$, and $w$, respectively) satisfy equations (7), (8), and (9). Because $r+s+w=1$, the steady-state value of $w$ satisfies

$$
(A B+1) w^{2}+A w-A=0,
$$

with $A=(1-\tau) / \tau$ and $B=\rho /(1-\rho)$. Equation (A17) has a unique solution $w(\tau) \in(0,1)$ for each $\tau \in(0,1)$. Furthermore, when inequality (A15) fails, there is a unique $\tau \in(0,1)$ for which $w(\tau)$ satisfies equation (A16). QED

Proof of proposition 5. Following the construction in the proof of proposition 4 , it follows that the value functions for a productive equilibrium without enforcement are given by

$$
V=(1-\delta)^{-1}(1-\delta \rho(1-w))^{-1}(a(1-\delta(1-w)))
$$

and

$$
W=(1-\delta)^{-1}(1-\delta \rho(1-w))^{-1} w a,
$$

which are derived from equations (A10) and (A11) by setting $c=0$.

An equilibrium with the desired characteristics exists if the two conditions in the statement of the proposition hold. By equation (A18),

$$
\frac{a}{b} \geq 1-\delta \rho\left(1-w^{*}(\rho)\right)
$$

implies that $V \geq b+\delta W$ (so that agents wish to cooperate in fresh relationships instead of deviating and joining the unmatched). By equation (A19),

$$
\frac{a}{l} \geq \frac{1-\delta \rho\left(1-w^{*}(\rho)\right)}{w^{*}(\rho)}
$$

implies that $W \geq \bar{l}$ (so that agents wish to leave stale relationships). QED

\section{References}

Allen, Franklin, Jun Qian, and Meijun Qian. 2005. Law, finance, and economic growth in China. Journal of Financial Economics 77, no. 1: 57-116.

Becker, Gary S. 1981. A treatise on the family. Cambridge, MA: Harvard University Press.

Binner, Jane M., and Antony W. Dnes. 2001. Marriage, divorce, and legal change: New evidence from England and Wales. Economic Inquiry 39: 298-306.

Carmichael, H. Lorne, and W. Bentley MacLeod. 1997. Gift giving and 
the evolution of cooperation. International Economic Review 38: 485-509.

Dixit, Avinash. 2003. On modes of economic governance. Econometrica 71, no. 2:449-81.

Friedberg, Leora. 1998. Did unilateral divorce raise divorce rates? Evidence from panel data. American Economic Review 88:608-27.

Greif, Avner. 1994. Cultural beliefs and the organization of society: A historical and theoretical reflection on collectivist and individualist societies. Journal of Political Economy 102:912-50.

Hosios, Arthur J. 1994. Unemployment and vacancies with sectoral shifts. American Economic Review 84:124-44.

Jovanovic, Boyan. 1979. Job matching and the theory of turnover. Journal of Political Economy 87, no. 5:972-90.

Kandori, Michihiro. 1992. Social norms and community enforcement. Review of Economic Studies 59:63-80.

Kranton, Rachel. 1996. The formation of cooperative relationships. Journal of Law, Economics, and Organization 12:214-33.

La Porta, Rafael, Florencio Lopez-de-Silanes, Cristian Pop-Eleches, and Andrei Shleifer. 1998. Law and finance. Journal of Political Economy 106:1113-55.

Lindsey, John, Ben Polak, and Richard Zeckhauser. 2001. Free love, fragile fidelity, and forgiveness: Rival social conventions under hidden information. Unpublished manuscript, Economics Department, Yale University.

McMillan, John, and Christopher Woodruff. 1999. Dispute prevention without courts in Vietnam. Journal of Law, Economics, and Organization 15:637-58.

- 2000a. Interfirm relationships and informal credit in Vietnam. Quarterly Journal of Economics 114:1285-1320.

- 2000b. Private order under dysfunctional public order. Michigan Law Review 98:2421-58.

Milgrom, Paul, Douglass North, and Barry Weingast. 1990. The role of institutions in the revival of trade: The medieval law merchant, private judges, and the champagne chairs. Economics and Politics 2:1-23.

Mortensen, Dale T., and Christopher A. Pissarides. 1994. Job creation and job destruction in the theory of unemployment. Review of Economic Studies 61:1138-51.

Ramey, Garey, and Joel Watson. 1997. Contractual fragility, job destruction, and business cycles. Quarterly Journal of Economics 112:873-911.

Sobel, Joel. 1985. A theory of credibility. Review of Economic Studies 52: 557-73.

Watson, Joel. 1999. Starting small and renegotiation. Journal of Economic Theory 85:52-90. 